\title{
Facing up to the reality of missed care
}

\section{Bonnie J Wakefield}

Correspondence to Bonnie J Wakefield,

Center for Comprehensive Access \& Delivery Research and Evaluation, lowa City Veterans Affairs Healthcare System, Sinclair School of Nursing, University of Missouri, Columbia, MO 65211, USA; wakefieldb@missouri.edu

Accepted 12 September 2013 Published Online First 18 November 2013

\section{CLinked}

- http://dx.doi.org/10.1136/ bmjqs-2012-001767 - http://dx.doi.org/10.1136/ bmjqs-2013-002318

To cite: Wakefield BJ. BMJ Qual Saf 2014;23:92-94.
Recent reports ${ }^{1} 2$ stemming from a large study in Europe explore the concept of 'missed care' by nurses. Missed care is defined as the omission of any aspect of required patient care. ${ }^{3}$ Beyond the expected finding that nurse-patient staffing ratios are associated with missed care, the work environment and registered nurses (RN) carrying out non-nursing tasks were also important predictors. ${ }^{1}$ The frequency of missed care was also associated with nurses' perceptions of patient safety. ${ }^{2}$ These findings are consistent with findings from a series of studies by Kalisch and colleagues in the USA. ${ }^{4} 5$ However, only recently has the issue of missed care been openly discussed in the literature. What are the implications of these findings?

Common activities are missed across hospitals and countries that have different national healthcare delivery systems. In these survey-based studies, nurses report prioritising direct patient care needs such as medication administration, but appear to assign lower priority to patient care interventions such as ambulation, psychosocial needs, care planning and patient education. The basis for these day-to-day, hour-to-hour, and in some cases minuteto-minute, decisions on what to prioritise is unknown, and poses extraordinarily rich questions for future research. Do nurses prioritise medications and treatments because they are formal physicians' orders? Do they place greater value on medications and treatments over other interventions needed to prepare the patient (and their informal care givers) for discharge? Is medication administration prioritised over other interventions because it addresses an immediate need, and is now becoming easily tracked through electronic medication administration records relative to other interventions such as ambulation or discharge planning, which may by their nature be somewhat more amorphous? Alternatively, do nurses prioritise medication administration more because of the critical role played by pharmaceutical interventions and their self-perceived competency in medication administration versus their feelings of ambiguity or perhaps less competence in offering psychosocial support, planning care or the discharge transition, and patient education?

In order to counteract low staffing and the resulting problem of missed care, some states and professional organisations in the USA have proposed mandated minimum staffing levels. There is controversy about this approach, as meeting the minimum may result in a 'race to the bottom' in staffing. Currently, some hospitals approach efficient staffing by analysing patient census and acuity every $4 \mathrm{~h}$ and adjusting staffing accordingly. However, it is not clear how these frequent staffing adjustments influence continuity of care and the potential for increased care problems, including missed care (due to ongoing prioritisation of what will and will not get done) due to multiple handoffs throughout the day. It is also unknown how frequent staffing adjustments affect the nursing practice environment, which is itself an important predictor of missed care.

There are also a number of potential questions concerning whether changes at the national system level in the financing, organisation and delivery of care may be increasingly forcing nurses to prioritise what will be left undone. Have hospitals stepped back to re-examine the question of what exactly needs to be done for patients by nurses, or are they continuing to presume that all the tasks that have been added to the nurses' responsibilities are essential and can be done with current nursing resources? With the increasing numbers and combinations of patient diagnoses and co-morbidities, has sufficient thought been given to not just the numbers but also the mix of nurses (eg, associate degree, BSN, advance practice nurses) needed to plan, organise, 
direct and actually provide the specific care and services needed?

In the studies reported, ${ }^{12}$ the quality of the nursing practice environment had the strongest relationship with missed care. The practice environment includes such things as staffing, resources, management and leadership, support from other nurses and physicians, and participation in decision making. Complexity compression is a recently identified concept related to the practice environment that may have implications for missed care. ${ }^{6}$ Complexity compression explains aspects of the practice environment not due solely to patient acuity, but rather the increasing complexity of and demand from systems and patients. Complexity compression has been defined as "what nurses experience when expected to assume additional, unplanned responsibilities while simultaneously conducting their multiple responsibilities in a condensed time frame". ${ }^{6}$ In their work, Krichbaum et al identified six themes associated with complexity compression: personal (eg, fatigue, family issues), environmental (eg, inadequate physical surroundings at work, conflict with coworkers, trust and respect), practice (eg, delegation, dilemmas posed by competing demands, prioritising multiple tasks within a time frame), systems (eg, system breakdowns, multiple organisation changes, documentation requirements, budgets, staffing, and regulatory requirements), administration and management (eg, inexperienced managers, lack of knowledge of what nurses do by administrators, numerous changes in upper management), and autonomy and control (eg, nursing input not sought about decisions which affect nursing practice at the unit level, or solicited input not valued). A study by Aiken and colleagues $^{7}$ showed that the beneficial effects associated with better staffing ratios are lost in places with poor work environments. Thus, while adjusting staffing ratios may seem like an easy fix, other critical aspects of the work environment likely affect the prevalence of missed care.

Of the six themes, the three most salient from the Krichbaum et al analysis were systems, practice and autonomy. One issue contributing to complexity compression is lack of knowledge of what nurses do. There may be a perception that, when additional tasks need to be accomplished, that 'the nurses can take care of it' because they are there 24/7. Often these tasks do not require the skills of a registered nurse (eg, checking the medication refrigerator thermometer). Moreover, new tasks for nurses are added frequently (eg, medication reconciliation, various risk assessments for falls, pressure ulcers and delirium), but rarely are other tasks simultaneously subtracted. To what extent is the input of nurses sought when making such decisions about workload, and which of these tasks are truly valueadded from the patient's perspective?

Ask a nurse what they do and the response will likely include a list of tasks that comprise their day at work.
Most nurses find it difficult to describe their roles. A number of nursing terminologies developed over the years have attempted to fill this void. Unfortunately, there is no general agreement on which terminology to use, and those in existence are sometimes awkward and time consuming to learn and use, adding to the existing documentation burden for nurses. Physicians have standardised terminologies and coding systems (eg, ICD, CPT) for capturing their practice as reflected in the patient record. These terminologies can be coded within the electronic health record (EHR) and retrieved for later analysis. Without an agreed-upon standardised terminology, it is difficult to accurately reflect nursing care in an EHR and then use the data to assess whether nursing care has been 'missed' and the extent to which missed care influences patient outcomes. Improving documentation systems for nurses through an efficient EHR may free up time so that nurses can decrease the amount of missed care, allowing the system to reflect the important contributions of nursing to patient outcomes. Such improvements may also increase nurses' morale, as many complain that they spend as much time documenting as they do interacting with patients or performing patient-related tasks.

Change is the only constant in healthcare over the past few decades. There will be continuing emphasis on reducing healthcare costs, improving efficiency, and decreasing waste, inappropriate interventions and non-evidence based care. Since the Institute of Medicine reports of To Err is Human and Crossing the Quality Chasm, there is and will continue to be increasing emphasis on quality, safety and patientcentred care. Simultaneously, as care continues to move to outpatient settings, the acuity of inpatients will increase. Thus increasing demands will be placed on nurses working in acute care environments to provide care in an efficient manner that is effective, high quality and safe.

As noted above, the findings of these studies raise a host of important questions urgently requiring additional research. Some important questions have begun to be addressed. To what extent is inpatient care a team activity? Who constitutes the team? Are the roles and expectations of the team members clearly identified and communicated? How well do team members communicate? Do inpatient staff accept the concept of working as a team? Kalisch and Lee found that lack of teamwork accounted for about $11 \%$ of missed nursing care. ${ }^{8}$ In an era of increased emphasis on patient-centred care, unfortunately patients also recognise missed care, and their perceptions are consistent with nurses' reports. ${ }^{9}$ Missed care has an effect on patient fall rates. ${ }^{4}$ Future work needs to address the effects on other short and long term patient outcomes. Missed care also directly affects nurses' satisfaction ${ }^{10}$ and turnover. ${ }^{11}$

Talk to any nurse who has worked in an acute care setting and ask them about missed care. Although it is 
rarely acknowledged or discussed, most nurses know it occurs. The question is, who will provide the leadership for nurses in their decisions about what to do or not do? As has often been said, 'every system is perfectly designed for the results it achieves'. The frequency of missed care is an unintended, but nonetheless predictable, consequence of our current systems of care. Unless we redesign these systems, including rethinking our approach to task assignment and nursing staff mix, we cannot expect this troubling problem to go away.

Disclaimer The content is solely the responsibility of the author and does not necessarily represent the position or policy of the Department of Veterans Affairs or the United States government.

\section{Competing interests None.}

Provenance and peer review Commissioned; internally peer reviewed.

\section{REFERENCES}

1 Ausserhofer D, Zander B, Busse R, et al. Prevalence, patterns and predictors of nursing care left undone in European hospitals: results from the multi-country cross-sectional RN4CAST study. BMJ Qual Saf 2014;23:126-135.
2 Ball JE, Murrells T, Rafferty AM, et al. 'Care left undone' during nursing shifts: associations with workload and perceived quality of care. BMJ Qual Saf 2014;23:116-125.

3 Kalisch BJ, Landstrom GL, Hinshaw AS. Missed nursing care: a concept analysis. J Adv Nurs 2009;65:1509-17.

4 Kalisch BJ, Tschannen D, Lee KH. Missed nursing care, staffing, and patient falls. J Nurs Care Qual 2012;27:6-12.

5 Kalisch BJ, Tschannen D, Lee H, et al. Hospital variation in missed nursing care. Am J Med Qual 2011;26:291-9.

6 Krichbaum K, Diemert C, Jacox L, et al. Complexity compression: nurses under fire. Nurs Forum 2007;42:86-94.

7 Aiken LH, Cimiotti JP, Sloane DM, et al. Effects of nurse staffing and nurse education on patient deaths in hospitals with different nurse work environments. Med Care 2011;49: 1047-53.

8 Kalisch BJ, Lee KH. The impact of teamwork on missed nursing care. Nurs Outlook 2010;58:233-41.

9 Kalisch BJ, McLaughlin M, Dabney BW. Patient perceptions of missed nursing care. Jt Comm J Qual Patient Saf 2012;38: 161-7.

10 Kalisch BJ, Tschannen D, Lee H. Does missed nursing care predict job satisfaction? J Healthc Manag 2011;56:117-31.

11 Tschannen D, Kalisch BJ, Lee KH. Missed nursing care: the impact on intention to leave and turnover. Can J Nurs Res 2010;42:22-39. 